\title{
Erratum: Testing the threshold expansion for three-particle energies at fourth order in $\phi^{4}$ theory [Phys. Rev. D 96, 054515 (2017)]
}

\author{
Stephen R. Sharpe \\ (Received 18 October 2018; published 8 November 2018)
}

DOI: 10.1103/PhysRevD.98.099901

I have found that a diagram is missing from Fig. 5 of the paper, and that the description of the analysis of Fig. 5(e) is partially incorrect. Nevertheless, as explained here, the final results of the paper are unaffected by these errors.

Figures 1(a) and 1(b) here reproduce Figs. 5(e) and 5(f) from the paper, while Fig. 1(c) shows the diagram that was missed. The discussion of Fig. 1(a) in Sec. III D 3 of the paper is correct except for one point - the counterterm contribution that is needed from Fig. 1(b) is not $A_{2 t}+A_{2 u}$, as stated in the paper, but instead is $A_{2 t}$. With this change, the diagram plus counterterm do not contribute to either $\partial_{\tau} C_{3, \text { thr,conn }}^{(4)}$ or $\mathcal{M}_{3 \text {,thr }}$, and thus an explicit calculation of the diagram is not needed. This conclusion is the same as that reached in the original paper.

Turning now to the new diagram, Fig. 1(c), this must be combined with the $A_{2 u}$ counterterm contribution from Fig. 1(b). The combination is finite in the ultraviolet (UV). The diagram has two three-particle cuts, and thus is potentially infrared (IR) divergent, but, as I explain below, this divergence is canceled by terms in the numerator. Because of this, the sums over both loop momenta in Fig. 1(c) can be converted to integrals, up to corrections of $\mathcal{O}\left(L^{-7}\right)$ that are not being considered. Using the general argument given in the paper, it follows that the contribution of this diagram to $\partial_{\tau} C_{3 \text {,thr }}^{(4)}(0)$ is of the standard form, Eq. (75) of the paper. Specifically, the diagram leads to a contribution to the energy shift proportional to $\mathcal{M}_{3 \text {,thr }}\left[\right.$ Fig. 1(c)] $/ L^{6}$, where $\mathcal{M}_{3 \text {,thr }}$ [Fig. 1(c)] is the result of evaluating the same diagram, but now interpreted as a contribution to $\mathcal{M}_{3 \text {,thr }}$. Thus, the diagram need not be explicitly calculated, and the key result of the paper, Eq. (141), remains valid.

I now return to the cancelation of the IR divergence in Fig. 1(c). I label the upper loop momentum $\vec{p}$ and the lower one $\vec{q}$. Note that, unlike for Fig. 1(a), the loops do not factorize, since the momentum $\vec{p}$ flows through the lower loop. The sum over $\vec{q}$ is UV and IR finite (after inclusion of the counterterm), and so can be converted to an integral, leading to a function $J\left(p^{2}\right)$, with $p^{2} \equiv \vec{p}^{2}$. In the renormalization scheme that I am using, the counterterm completely cancels the loop contribution when $\vec{p}=0$, implying, for small $\vec{p}$, that $J\left(p^{2}\right) \propto p^{2}$. The sum over $\vec{p}$ has the form $\sum_{\vec{p}} J\left(p^{2}\right) / p^{4}$, due to the presence of two three-particle cuts. The form of $J$ converts this to the sum $\sum_{\vec{p}} 1 / p^{2}$, which can be converted to an IR convergent integral up to corrections that contribute to the energy at $O\left(L^{-7}\right)$.

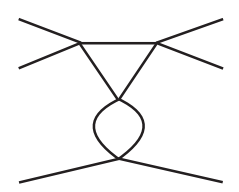

(a)

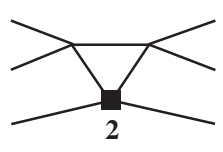

(b)

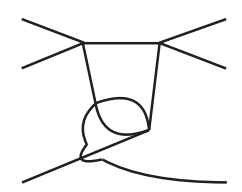

(c)

FIG. 1. Diagrams discussed in the text.

This error was discovered while studying the contribution to the threshold energy shift from the $d$-wave scattering amplitude [1] in a numerical implementation of the quantization condition of Ref. [2]. The dependence found numerically cannot arise from any of the diagrams considered in the paper, but can arise from the missing diagram.

[1] T. D. Blanton, F. Romero-López, and S. R. Sharpe (to be published).

[2] M. T. Hansen and S. R. Sharpe, Phys. Rev. D 90, 116003 (2014). 